\title{
Moluscos marinos intermareales y submareales entre la Boca del Guafo y el estero Elefantes, sur de Chile
}

\author{
Cecilia Osorio $^{1}$ \& David G. Reid ${ }^{2}$ \\ ${ }^{1}$ Departamento de Ciencias Ecológicas, Facultad de Ciencias, \\ Universidad de Chile. Casilla 653, Santiago, Chile \\ ${ }^{2}$ Department of Zoology, The Natural History Museum. London SW7 5BD, United Kingdom
}

\begin{abstract}
RESUMEN. Se analizan los moluscos marinos recolectados en los canales Jacaf y Puyuhuapi, seno de Aysén y esteros Quitralco y Cupquelán, en el área de fiordos del sur de Chile. Las muestras se obtuvieron durante el crucero de investigación Cimar 7 Fiordos, realizado entre el 8 y 20 de julio de 2001. La recolección de muestras bentónicas se realizó entre la Boca del Guafo y estero Elefantes. Las muestras se obtuvieron manualmente y por medio de una rastra Agassiz en 20 estaciones a profundidades que variaron entre 0 y $330 \mathrm{~m}$. Los resultados de este estudio permitieron reconocer 22 especies (1 poliplacóforo, 7 gastrópodos, 12 bivalvos y 2 escafópodos). Se entrega la descripción y la fotografía de 19 de ellas. Para cada especie se indican nuevos datos del hábitat y su distribución geográfica. Se reconocieron siete especies como nuevos registros geográficos: Calliostoma nudiusculum, Calliostoma consimilis, Capulus compressus, Falsilunatia patagonica, Aeneator fontainei, Fissidentalium majorinum y Rhabus perceptum, seis de ellas vinculadas con la malacofauna magallánica y solo Aeneator fontainei fue registrado anteriormente en la costa norte de Chile.
\end{abstract}

Palabras clave: moluscos marinos, intermareal, submareal, fiordos, Chile.

\section{Intertidal and subtidal marine molluscs between Boca del Guafo and Elefantes Fjord, southern Chile}

\begin{abstract}
A study was made of marine molluscs collected in the fjord region of southern Chile, including the Jacaf and Puyuhuapi channels, Aysen Sound and the Quitralco and Cupquelan fjords. Samples were obtained during the Cimar 7- Fjords research cruise, of 8-20 July 2001. Collections of benthic samples were made between Boca del Guafo and the Elefantes fjord. Samples were taken by hand with an Agassiz trawl from 20 stations at various depths between 0 and $330 \mathrm{~m}$. A total of 22 species were recorded (1 polyplacophoran, 7 gastropod, 12 bivalve and 2 scaphopod). Of these, 19 are described and illustrated, together with new information on habitat and geographical distribution. We report new geographical records for the following seven species: Calliostoma nudiusculum, Calliostoma consimilis, Capulus compressus, Falsilunatia patagonica, Aeneator fontainei, Fissidentalium majorinum and Rhabdus perceptum, six of these are northward extensions of the magellanic molluscan fauna while the record of Aenaeator fontainei is a new southern limit.
\end{abstract}

Key words: marine molluscs, intertidal, subtidal, fjords, Chile. 


\section{INTRODUCCIÓN}

La zona de fiordos y canales del sur de Chile es históricamente una región de escasa intervención humana, con lejanos centros poblados, alta pluviosidad, difíciles condiciones meteorológicas para la navegación y gran riqueza faunística. Sin embargo, hasta principios de la década del 90, era una de las áreas geográficas de Chile menos estudiada desde el punto de vista oceanográfico y de la biodiversidad marina.

En la última década la creciente demanda de uso del borde costero y de sus aguas, principalmente para actividades de acuicultura, pesquería y turismo, motivaron al Comité Oceanográfico Nacional iniciar, en 1995, un programa de investigación científico-marina a esta vasta zona del territorio nacional, que se denominó programa Cimar el cual se concibió como una serie de campañas a lugares remotos escasamente estudiados. Los cruceros, realizados entre 1995 y 1998, consistieron en investigaciones de tipo exploratorias, en las que se abarcó, en etapas sucesivas, la mayor parte de las aguas interiores entre Puerto Montt y Cabo de Hornos.

Los resultados han generado un significativo avance en el conocimiento de la geomorfología de los fiordos (Araya-Vergara, 1997, 1999), de las características físicas y químicas de la columna de agua (Silva et al., 1997; Silva \& Calvete, 2002), de la oceanografía de los estuarios (Basten \& Clement, 1999); de sedimentos (Bonert, 1997; Bonert \& Estrada, 2001; Silva et al., 2001; Silva \& Ortiz, 2002; Pineda et al., 2002), de patrones generales de circulación (Fierro et al., 2000), de diversidad biológica de plancton (Avaria et al., 1997; Palma \& Rosales, 1997; Mujica \& Medina, 1997; Balbontín \& Bernal, 1997; Avaria et al., 1999; Palma et al., 1999; Bernal \& Balbontín, 1999; Mujica \& Medina, 2000; Vega et al., 2000; Marín \& Delgado, 2001), de peces (Pequeño \& Lamilla, 1997; Pequeño, 1999, 2000) y de crustáceos decápodos (Retamal \& Gorny, 2001).

Otros antecedentes sobre biodiversidad marina en el área de estudio generados por diferentes expediciones científicas realizadas durante los dos siglos pasados y, los aportes de diversos científicos participantes en las expediciones Raleigh, se resumen en Osorio et al. (2002). Con respecto a los moluscos intermareales, el estudio de Reid \& Osorio (2000) entrega un completo análisis de la historia de los estudios malacológicos en Chile y específicamente del área de estudio.
Durante el año 2001, se realizó el crucero Cimar 7 Fiordos compuesto por dos campañas, efectuadas en invierno y primavera. De un total de 17 proyectos aprobados, uno de ellos fue el estudio de la diversidad de moluscos bentónicos de los fiordos del sur de Chile, colectados entre la boca del Guafo y estero Elefantes. El objetivo de este estudio es incrementar el conocimiento de la distribución, hábitat e identificación de los moluscos marinos colectados, destacando los nuevos registros geográficos, para el área de los fiordos. Esta contribución es de interés zoológico y malacológico y su valor se incrementará al considerar que puede servir de base a futuros estudios.

\section{MATERIALES Y MÉTODOS}

Las muestras bentónicas se obtuvieron entre el 8 y 20 de julio de 2001 abordo del AGOR "Vidal Gormaz", desde la boca del Guafo (4339'18"S) hasta el estero Elefantes $\left(46^{\circ} 28^{\prime} 88^{\prime \prime} \mathrm{S}\right)$. Se obtuvo material de los sectores seno Soto, canal Jacaf, canal Puyuhuapi, seno Aysén, estero Queulat, estero Pangal, estero Cupquelán, seno Magdalena y bahía Exploradores (Fig. 1).

Los especímenes se recolectaron mediante una rastra Agassiz ( $1 \mathrm{~cm}$ de abertura de malla) en 20 estaciones, a profundidades entre 20 y $330 \mathrm{~m}$. Además, se efectuaron 3 estaciones ( $\mathrm{A}, \mathrm{G}$ y $\mathrm{N}$ ) desde el intermareal hasta los $5 \mathrm{~m}$ de profundidad, por medio de buceo autónomo. Los datos de salinidad y temperatura se obtuvieron mediante CTD (SBE-25), a las profundidades indicadas en la Tabla 1. Los ejemplares se fijaron en una solución de formalina al $10 \%$ con agua de mar. Para la identificación se trabajó al máximo nivel taxonómico posible mediante bibliografía especializada y se comparó parte del material con los tipos depositados en las colecciones del Natural History Museum de Londres. Las especies que corresponden a nuevos registros geográficos y otros moluscos que no se incluyeron en el trabajo de Reid \& Osorio (2000), se fotografiaron en el Natural History British Museum.

Se entrega la descripción sistemática de 22 especies, con la referencia de la descripción original, una o varias referencias de los más recientes estudios taxonómicos, los que pueden ser consultados por completas sinonimias. La descripción y dimensiones de las conchas están basadas en el material recolectado en este estudio. Las medidas controladas de longitud en gastrópodos son tomadas desde 


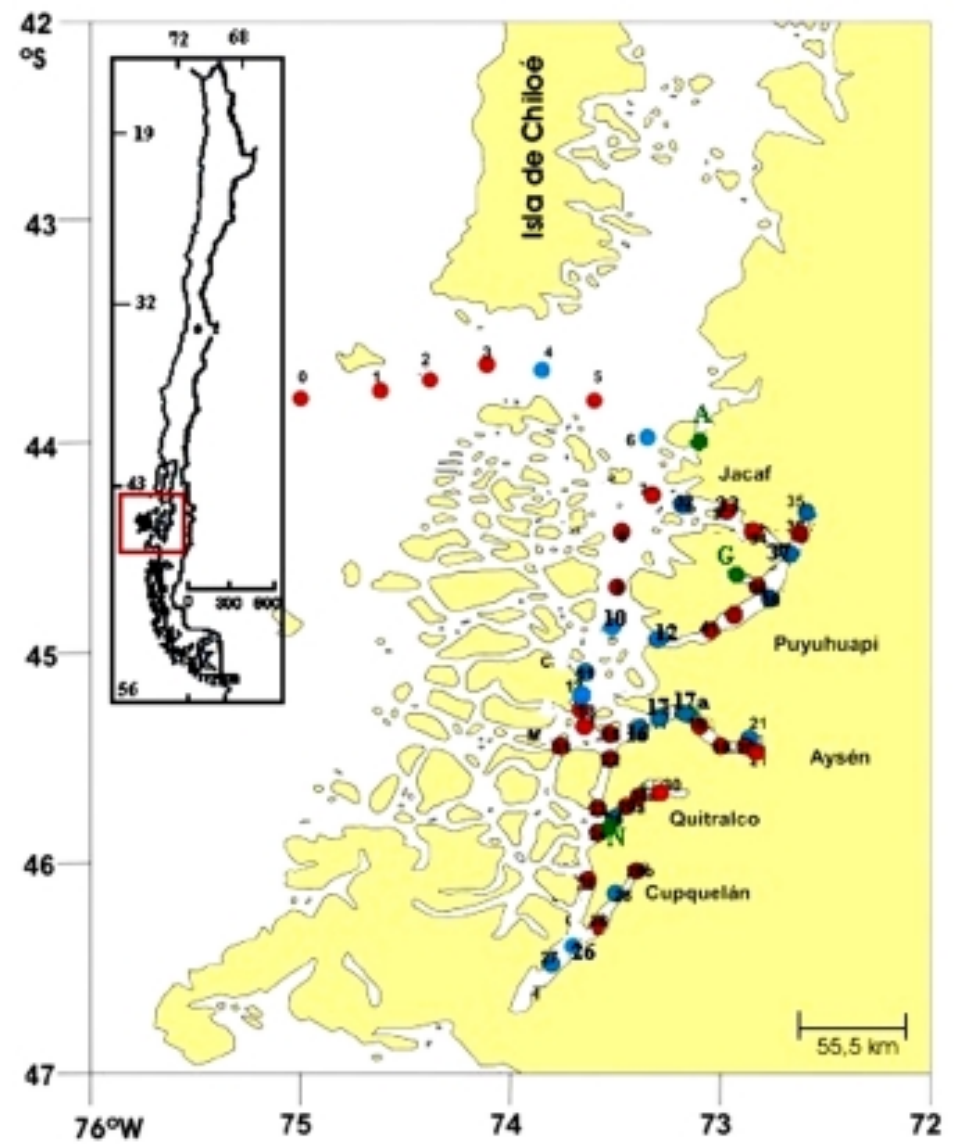

Figura 1. Estaciones de muestreo (rojo), en azul los registros de moluscos y en verde las estaciones del intermareal (A, G y N).

Figure 1. Sampling stations (red); those with molluses in blue (subtidal) and green: A, G and N (intertidal).

el ápice a la base, en los bivalvos desde el borde del extremo anterior al posterior. Se incluye la fotografía de 19 de las 22 especies identificadas, ya que tres de ellas tenían el material erosionado, o con epibiontes, o por estar encogidos o doblados como en el caso de nudibranquios.

\section{RESULTADOS}

\section{DESCRIPCIÓN SISTEMÁTICA}

\section{POLYPLACOPHORA CHITONIDAE}

Tonicia disjuncta (Frembly, 1827) (Fig. 1a)

Chiton disjunctus Frembly, 1827: 203, suppl. lám. 17, fig. 5 .
Tonicia disjuncta (Leloup, 1956): 67-68, figs. 36, 37 (sinonimia); Kaas \& Van Belle, 1998: 63; Forcelli, 2000: 186, fig. 627; Slieker, 2000: 78, fig. 121.

Descripción: placas planas sin imbricarse una a otras (de ahí su nombre). Sin umbos salientes ni notorias áreas laterales. Láminas suturales fuertes y grandes. Con un seno amplio y denticulación notoria. Color pardo verdoso o café rojizo con marcas verdosas y con líneas de crecimiento en todas las placas. Se destacan ojos extra pigmentados. Cinturón ancho de color rojizo. La talla máxima fue de $94 \mathrm{~mm}$ de longitud.

Hábitat: $T$. disjuncta se recolectó desde el intermareal hasta $5 \mathrm{~m}$ de profundidad. Datos anteriores indican que vive sobre sustrato rocoso desde el intermareal a $8 \mathrm{~m}$ de profundidad (Slieker, 2000). 
Tabla 1. Lista de las estaciones donde fueron recolectados los moluscos inter y submareales entre la boca del Guafo y el estero Elefantes. Crucero Cimar 7 Fiordos.

Table. 1. List of stations where intertidal and subtidal molluses were collected, between Boca del Guafo and Elefantes Fjord. Cimar 7 Fiordos Cruise.

\begin{tabular}{|c|c|c|c|c|c|}
\hline Estación & Localidad & Latitud ( $\left.{ }^{\circ} \mathrm{S}\right)$ & Longitud $\left({ }^{\circ} \mathrm{W}\right)$ & Fecha & Profundidad (m) \\
\hline 4 & Boca Guafo & $43^{\circ} 39^{\prime} 18^{\prime \prime}$ & $73^{\circ} 50^{\prime} 95^{\prime \prime}$ & 08/07/2001 & 150 \\
\hline 6 & Moraleda & $43^{\circ} 59^{\prime} 19^{\prime \prime}$ & $73^{\circ} 21^{\prime} 87^{\prime \prime}$ & 09/07/2001 & 120 \\
\hline 10 & Moraleda & $44^{\circ} 53^{\prime} 38^{\prime \prime}$ & $73^{\circ} 31^{\prime} 25^{\prime \prime}$ & 09/07/2001 & 140 \\
\hline 12 & Moraleda & $45^{\circ} 12^{\prime} 29^{\prime \prime}$ & $73^{\circ} 39^{\prime} 75^{\prime \prime}$ & 09/07/2001 & 140 \\
\hline 13 & Moraleda & $45^{\circ} 16^{\prime} 17^{\prime \prime}$ & $73^{\circ} 39^{\prime} 98^{\prime \prime}$ & 09/07/2001 & 50 \\
\hline 14 & Moraleda & $45^{\circ} 21^{\prime} 10^{\prime \prime}$ & $73^{\circ} 38^{\prime} 88^{\prime \prime}$ & 09/07/2001 & 140 \\
\hline 16 & Aysén & $45^{\circ} 21^{\prime} 80^{\prime \prime}$ & $73^{\circ} 22^{\prime} 78^{\prime \prime}$ & 09/07/2001 & 200 \\
\hline 17 & Aysén & $45^{\circ} 18^{\prime} 58^{\prime \prime}$ & $73^{\circ} 17^{\prime} 51^{\prime \prime}$ & $10 / 07 / 2001$ & 125 \\
\hline 18 & Aysén & $45^{\circ} 21^{\prime} 00^{\prime \prime}$ & $73^{\circ} 05^{\prime} 95^{\prime \prime}$ & $10 / 07 / 2001$ & 300 \\
\hline 20 & Aysén & $45^{\circ} 26^{\prime} 36^{\prime \prime}$ & $72^{\circ} 53^{\prime} 56^{\prime \prime}$ & $10 / 07 / 2001$ & 150 \\
\hline 21 & Aysén & $45^{\circ} 24^{\prime} 69^{\prime \prime}$ & $72^{\circ} 51^{\prime} 64^{\prime \prime}$ & $11 / 07 / 2001$ & 140 \\
\hline 22 & Costa & $45^{\circ} 29^{\prime} 56^{\prime \prime}$ & $73^{\circ} 31^{\prime} 03^{\prime \prime}$ & $10 / 07 / 2001$ & 250 \\
\hline 25 & Elefantes & $46^{\circ} 05^{\prime} 01^{\prime \prime}$ & $73^{\circ} 37^{\prime} 82^{\prime \prime}$ & $14 / 07 / 2001$ & 35 \\
\hline 26 & Elefantes & $46^{\circ} 19^{\prime} 69^{\prime \prime}$ & $73^{\circ} 42^{\prime} 88^{\prime \prime}$ & $14 / 07 / 2001$ & 12 \\
\hline 27 & Elefantes & $46^{\circ} 28^{\prime} 88^{\prime \prime}$ & $73^{\circ} 48^{\prime} 32^{\prime \prime}$ & $15 / 07 / 2001$ & 90 \\
\hline 29 & Quitralco & $45^{\circ} 46^{\prime} 85^{\prime \prime}$ & $73^{\circ} 30^{\prime} 60^{\prime \prime}$ & $13 / 07 / 2001$ & 90 \\
\hline 32 & Jacaf & $44^{\circ} 17^{\prime} 76^{\prime \prime}$ & $73^{\circ} 10^{\prime} 78^{\prime \prime}$ & $19 / 07 / 2001$ & 400 \\
\hline 35 & Ventisquero & $44^{\circ} 21^{\prime} 37^{\prime \prime}$ & $72^{\circ} 34^{\prime} 82^{\prime \prime}$ & $18 / 07 / 2001$ & 40 \\
\hline 37 & Ventisquero & $44^{\circ} 31^{\prime} 85^{\prime \prime}$ & $72^{\circ} 40^{\prime} 09^{\prime \prime}$ & $19 / 07 / 2001$ & 225 \\
\hline 39 & Puyuhuapi & $44^{\circ} 43^{\prime} 59^{\prime \prime}$ & $72^{\circ} 43^{\prime} 30^{\prime \prime}$ & $18 / 07 / 2001$ & 150 \\
\hline A & Melimoyu & $44^{\circ} 04^{\prime} 00^{\prime \prime}$ & $73^{\circ} 10^{\prime} 00^{\prime \prime}$ & $20 / 07 / 2001$ & $1 *$ \\
\hline G & Seno Magdalena & $44^{\circ} 41^{\prime} 00^{\prime \prime}$ & $72^{\circ} 50^{\prime} 00^{\prime \prime}$ & $18 / 07 / 2001$ & $3 *$ \\
\hline $\mathrm{N}$ & Estero Quitralco & $45^{\circ} 39^{\prime} 00^{\prime \prime}$ & $73^{\circ} 13^{\prime} 50^{\prime \prime}$ & $16 / 07 / 2001$ & $2 *$ \\
\hline
\end{tabular}

*estaciones intermareales

Distribución geográfica: se recolectó sólo en la estación 18. La bibliografía indica que la especie se distribuye entre Tumbes (Perú), Valparaíso y Ancud (Leloup, 1956), recientemente Slieker (2000) lo menciona en Concepción, Lirquén y de los $33^{\circ}$ a $49^{\circ} \mathrm{S}$.

Observaciones: especie poco común. El ejemplar fotografiado es de tamaño mayor $(94 \mathrm{~mm})$ al registrado por Castellanos (1988) de $67 \mathrm{~mm}$.

\section{GASTROPODA TROCHIDAE}

\section{Calliostoma nudiusculum (von Martens, 1881)}

Trochus (Margarita) nudiusculum von Martens, 1881: 131, lám.5, fig. 26.

Calliostoma nudiusculum (Carcelles, 1950): 54;
Carcelles \& Williamson, 1951: 264; Castellanos, 1989: 7-8, lám. 1, fíg. 2, lám. 3, fig. 1; Forcelli, 2000: 60, fig. 82; Linse, 2002: 75-76, lám. 4b.

Descripción: levemente más alto que ancho. Vueltas y base convexas. Ápice liso, amarillento, con protoconcha persistente, globosa. Color blanco iridiscente. El callo es blanco y no tapa el ombligo, que no está perforado. La escultura es notoriamente acordonada, el cordón anterior a la sutura es liso y los otros con nódulos pequeños, la última vuelta tiene seis cordones granulosos y dos lisos en el perímetro que se continúan lisos en la base. La base con finas estrías espirales. La abertura es subcircular con el borde ondulado por los cordones exteriores. Mide $11,5 \mathrm{~mm}$ longitud y $10,5 \mathrm{~mm}$ ancho.

Hábitat: se encontró a $106 \mathrm{~m}$ de profundidad, sobre fondo de arena, junto con Tritonia sp., Natica patagonica y Zygochlamys patagonica. En la loca- 
lidad tipo, Patagonia, se recolectó a 111 m de profundidad.

Distribución geográfica: sólo un ejemplar fue registrado en la estación 4. Anteriormente ha sido reconocida a los $43^{\circ} \mathrm{S}$ (Carcelles \& Williamson, 1951). Desde $\operatorname{los} 36^{\circ}$ a $54^{\circ} \mathrm{S}$ y desde $55^{\circ} 53^{\prime}$ hasta $60^{\circ} 23^{\prime} \mathrm{W}$ en la costa argentina (Castellanos, 1989). Este registro extiende hacia el norte la distribución latitudinal de la especie por la costa del Pacífico.

Observaciones: la descripción y dibujos entregados por Castellanos (1989) corresponde al ejemplar recolectado, pero hay claras diferencias con la descripción del color y de los cordones espirales con nódulos de Forcelli (2000). Posiblemente es una especie con una gran variabilidad.

\section{Calliostoma consimilis (E.A. Smith, 1881)} (Fig. 2c)

Trochus consimilis E.A. Smith, 1881: 34, lám. 4, fig. 11 .

Calliostoma consimilis (Strebel, 1905): 123, lám. 5, fig. 21; Carcelles, 1950: 53, lám.1; Castellanos 1967: 28, lám. 2, fig. 2; Forcelli, 2000: 59, fig. 75; Linse, 2002: 72-73, lám. 3b.

Descripción: concha trocoide con 7 vueltas rectas. Protoconcha lisa, blanca. Cada vuelta con 3 a 4 cordones fuertes, el primer cordón con gránulos notorios, sobresalientes. Sutura poco evidente. Ultima vuelta angulosa con 3 ó 4 surcos hasta el área del borde y otros 2 ó 3 cordones circundando el ombligo. Color violeta. Abertura oblicua cuadrangular, callo de color blanco. La talla máxima controlada fue de $8,3 \mathrm{~mm}$.

Hábitat: en este estudio se encontraron ejemplares entre 20 y $160 \mathrm{~m}$ en fondos de arena, junto a los braquiópodos Novocrania lecointei (Joubin, 1901) y juveniles de Macrandrevia sp. Castellanos (1967) indica que el crucero científico francés "Gazelle" la registró a $80 \mathrm{~m}$, y el "Alert" en el intermareal.

Distribución geográfica: se encontraron ejemplares en las estaciones 13,14 y 26. Registros anteriores indican que se distribuye entre los $30^{\circ} 43^{\prime} \mathrm{y}$ 52³6'S por el océano Atlántico a Tierra del Fuego y canal Smith (Castellanos, 1967; Castellanos \& Landoni, 1989). El presente registro extiende hacia la costa del Pacífico la distribución de la especie.

Observaciones: la especie es poco común. El ejemplar tipo tiene una talla de $10 \mathrm{~mm}$. El dibujo de Castellanos (1989), caracteriza muy bien la especie y los ejemplares analizados. La identificación fue confirmada por comparación con los tipos (BMNH 1879. 10. 15.29-34).

Falsimargarita sp. (Fig. 2b)

Descripción: concha muy delgada, con cinco vueltas convexas con cuatro cordones espirales notorios, lisos. Con la protoconcha sobresaliente redondeada. La línea de sutura definida. El color de la concha es blanco o iridiscente exterior e interiormente. Umbilico profundo y estrecho. La talla es de 8,0 $\mathrm{mm}$ de ancho y 9,1 $\mathrm{mm}$ de alto.

Hábitat: la muestra fue recolectada a $76 \mathrm{~m}$ de profundidad junto a Novocrania lecointei.

Distribución geográfica: el ejemplar se registró en la estación 13.

Observaciones: con un sólo ejemplar de Falsimargarita no fue posible la identificación a nivel de especie. Esta especie se parece a F. iris (E.A. Smith, 1915), pero tiene nueve costillas espirales sobre la penúltima vuelta, mientras el ejemplar de este estudio tiene sólo cuatro.

\section{CAPULIDAE}

\section{Capulus compressus E.A. Smith, 1891}

(Fig. 2e)

Capulus compressus E.A. Smith, 1891: 427, lám. 34, fig. 4; Carcelles, 1944: 4, fig. 2; 1950: 57; Castellanos, 1967: 54, lám. 3, fig. 5; Forcelli, 2000: 75, fig. 164 .

Descripción: concha muy frágil, capuliforme, alta, angosta, y comprimida lateralmente, plana a los lados. Ápice sobresaliente, con 2 ó 3 vueltas, a veces poco visibles y girada a la derecha. Con estriación concéntrica fina e irregular. Abertura oblonga, angosta, o elíptica de borde irregular. Color blanco. La talla máxima fue de $6,3 \mathrm{~mm}$.

Hábitat: en este estudio se encontró a 76 m de profundidad, junto al braquiópodo Novocrania lecointei $\mathrm{y}$ los moluscos Calliostoma consimilis y Zygochlamys patagonica. Anteriormente, se indicó que vive en fondos pedregosos con elementos faunísticos magallánicos, como Labidiaster sp., Gorgonocephalus sp., Cirolana sp., Eurypodius sp. y Calyptraea sp. (Carcelles, 1944). Epizoica o sobre fondos pedregosos sobre superficies duras. $\mathrm{Ob}-$ servaciones de la expedición Challenger lo registra 


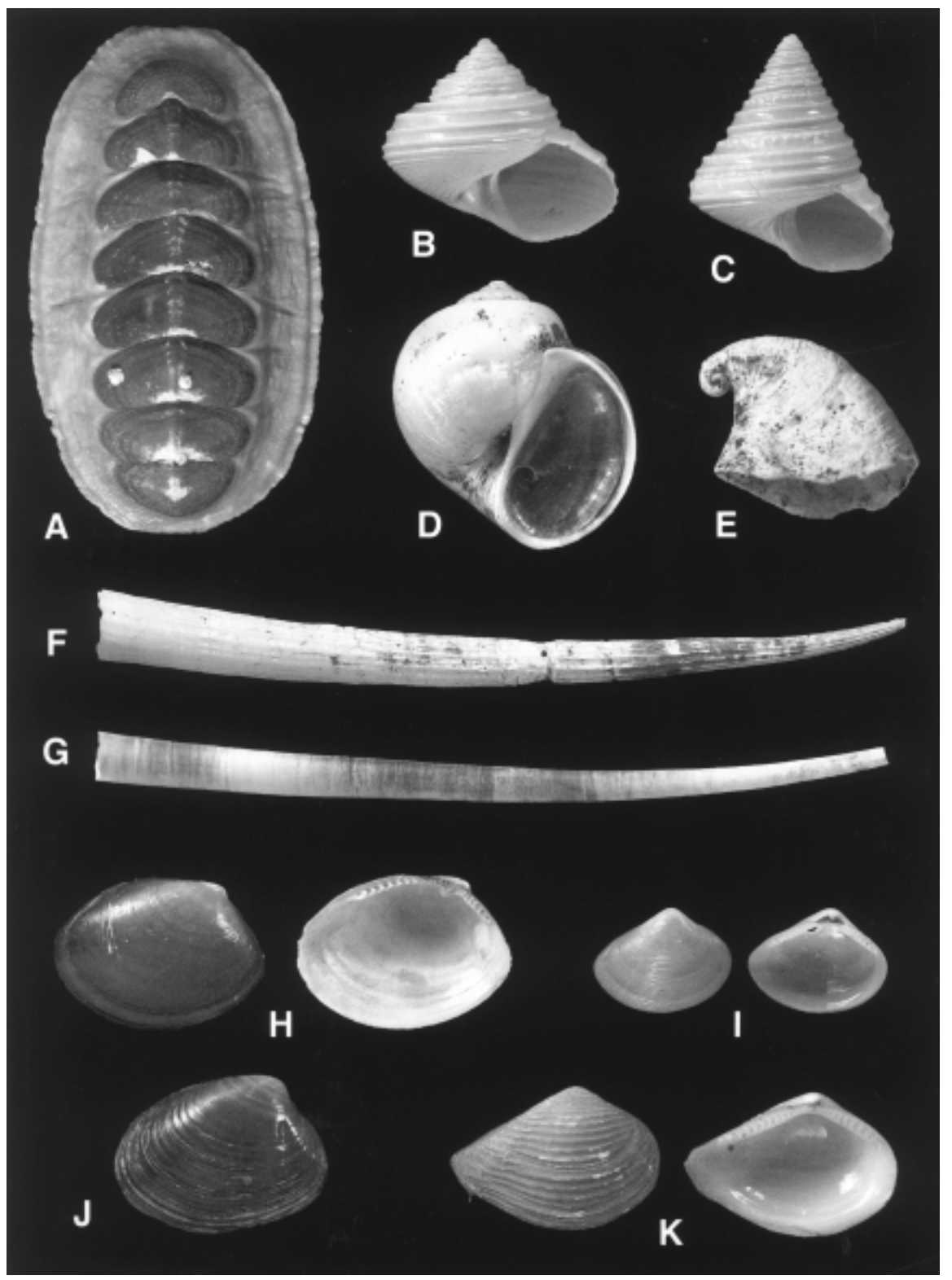

Figura 2. Lista de especies fotografiadas. a) Tonicia disjuncta (Frembly, 1827), estación 18,94 mm, b) Falsimargarita sp., estación 13, 9,0 mm, c) Calliostoma consimilis (E.A. Smith, 1881), estación 26, 8,3 mm, d) Falsilunatia patagonica (Philippi, 1847), estación 37, 14,0 mm e) Capulus compressus E.A. Smith, 1891, estación 13, 6,3 mm, f) Fissidentalium majorinum Mabille \& Rochebrune, 1889, estación 22, 36 mm, g) Rhabdus perceptum (Mabille \& Rochebrune, 1889), estación 22, 43 mm, h) Ennucula grayi (D’Orbigny, 1846), estación 21, 14,6 mm, i) Tindaria virens (Dall, 1889), estación 37, 4,7 mm, j) Ennucula puelcha (D’Orbigny, 1842), estación 21, 14,7 mm, k) Tindaria striata (King \& Broderip, 1832), estación 14, 8,8 mm.

Figure 2. List of species photographied. a) Tonicia disjuncta (Frembly, 1827), station 18, 94 mm, b) Falsimargarita sp., station 13, $9.0 \mathrm{~mm}$, c) Calliostoma consimilis (E.A. Smith, 1881), station 26, $8.3 \mathrm{~mm}$, d) Falsilunatia patagonica (Philippi, 1847), station 37, 14.0 mm, e) Capulus compressus E.A. Smith, 1891, station 13, 6.3 mm, f) Fissidentalium majorinum Mabille \& Rochebrune, 1889, station 22, $36 \mathrm{~mm}, \mathrm{~g}$ ) Rhabdus perceptum (Mabille \& Rochebrune, 1889), station 22, $43 \mathrm{~mm}$, h) Ennucula grayi (D'Orbigny, 1846), station 21, $14.6 \mathrm{~mm}$, i) Tindaria virens (Dall, 1889), station 37, $4.7 \mathrm{~mm}$, j) Ennucula puelcha (D'Orbigny, 1842), station 21, $14.7 \mathrm{~mm}$, k) Tindaria striata (King \& Broderip, 1832), station 14, $8.8 \mathrm{~mm}$. 
a $409 \mathrm{~m}\left(52^{\circ} 45^{\prime} \mathrm{S}, 73^{\circ} 45^{\prime} \mathrm{W}\right)$ y en Comodoro Rivadavia a $110 \mathrm{~m}$ (Castellanos, 1967).

Distribución geográfica: en este estudio se registraron dos ejemplares en la estación 13. Anteriormente, fue descrito para la costa Atlántica entre los 28 31'S y 55'42'S hasta el estrecho de Magallanes (Castellanos, 1967). El presente registro extiende su distribución geográfica hacia el norte de la costa Pacífica.

Observaciones: se registraron 2 ejemplares muertos de Capulus compressus, pero con sus conchas en buenas condiciones. Dell (1964a) dice que especies del género Capulus en varias partes del mundo son parásitos o semi-parásitos de pectínidos. Castellanos (1967) indica para esta especie una longitud máxima de $12,5 \mathrm{~mm}$, tamaño mayor al registrado en estos ejemplares.

\section{NATICIDAE}

\section{Falsilunatia patagonica (Philippi, 1845)}

(Fig. 2d)

Natica patagonica Philippi, 1845: 65.

Polinices patagonicus (Linse, 2002): 95-96, lám. 11.

Falsilunatia patagonica (Dell, 1990): 148 (sinonimia), fig. 248; Forcelli, 2000: 77, fig. 172.

Falsilunatia soluta (Gould, 1847): Castellanos 1990: 22, lám. 3, figs. 25-28.

Descripción: concha de tamaño pequeño, globosa, sólida o gruesa con la espira corta. La última vuelta muy grande. La línea de la sutura angosta y profunda. Exteriormente es lisa sin esculturas, sólo finas líneas de crecimiento. Umbilico grande y profundo, parcialmente tapado por el labio del callo de la columela. La abertura es oval. El color es blanco cubierto por un periostraco oliva pálido muy delgado que fácilmente se desprende. Talla máxima de $14 \mathrm{~mm}$.

Hábitat: se capturó entre los 106 a 330 m de profundidad. Un ejemplar fue recolectado por el Eltanin (Dell, 1990) cerca de las islas Malvinas entre 40$172 \mathrm{~m}$ de profundidad. Powell (1951) lo registró a 401-411 m de profundidad.

Distribución geográfica: los ejemplares se recolectaron en las estaciones 4, 14, 17a, 22, 32 y 37. Anteriormente, fue registrado entre el estrecho de Magallanes, Tierra del Fuego, e islas Malvinas hasta los $37^{\circ} \mathrm{S}$ (Castellanos, 1990). Powell (1951) indi- ca esta especie para los alrededores de la isla Georgia del Sur. Este estudio extiende la distribución geográfica de la especie hacia el norte por la costa del océano Pacífico.

Observaciones: el tamaño de los ejemplares varió entre 3,4 x 3,3 y 14 x 12,7 mm. Además, tanto la descripción como las fotografías de Falsilunatia soluta realizadas por Castellanos (1990) corresponden a esta especie, por lo cual se ha agregado este nombre a la sinominia.

\section{BUCCINIDAE}

\section{Aeneator fontainei (D’Orbigny, 1841)}

Fusus fontainei D’Orbigny, 1841: 447, lám. 63, fig. 2. Aeneator fontainei (McLean \& Andrade, 1982): 1314, fig. 31-39 (sinonimia).

Descripción: concha grande y fusiforme. La longitud de la abertura y el canal sifonal son más de mitad de la longitud total. La espira tiene 6 vueltas convexas con un área cóncava próxima a la sutura. La sutura es un fino surco angosto, nítidamente impreso. Presenta una escultura axial con 15 a 17 costillas redondeadas, alzadas hasta la penúltima vuelta, esta escultura falta en la última vuelta. La escultura espiral presenta 4 cordones mayores, angostos, poco alzados y de color café oscuro o café rojizo en las primeras vueltas. A veces aparecen cordones menores en los interespacios, algunos de color café oscuro. La superficie de la concha presenta un periostraco delgado de color café verdoso que fácilmente se desprende. El borde de la abertura es cortante y lirado o festoneado por las terminaciones de los numerosos cordones espirales externos. La talla máxima fue de $10,27 \mathrm{~cm}$.

Hábitat: un ejemplar se registró a 60 m de profundidad. Anteriormente se ha descrito para la especie un rango de profundidad de 20-350 m (McLean \& Andrade, 1982).

Distribución geográfica: en este estudio se recolectó en la estación 25. Se ha registrado anteriormente entre bahía Independencia, Perú a punta Toro, Chile (331'S) (McLean \& Andrade, 1982). Este trabajo extiende la distribución geográfica de la especie hacia el sur por la costa del océano Pacífico.

Observaciones: el ejemplar, vivo, presentaba sobre la concha tres hileras de cápsulas con un total de 24 cápsulas, 11 de ellas con juveniles intracapsulares. 


\section{DISCODORIDIDAE}

\section{Diaulula punctuolata (D'Orbigny, 1837)}

Doris punctuolata D’Orbigny, 1837: 186-187, lám. 16, figs. 4-6.

Anisodoris punctuolata (Schrödl, 1996): 25 (sinonimia), lám. 3, fig. 20.

Descripción: cuerpo elevado de color blanquecino a amarillento, a menudo con dos filas de 3-5 puntos pálidos a muy oscuros entre los rinóforos y branquias. Dorso cubierto densamente por cariofilídeos delgados de tamaños más o menos iguales. Los rinóforos poseen más de 15 hojas y están circundados de pliegues en forma de collar elevado y cubierto por cariofilídeos. Tentáculos digitiformes. Pie ancho, surcado por dos labios. El borde del manto tiende a la autonomía. Largo total hasta $10 \mathrm{~cm}$.

Hábitat: En este estudio se registró a 60 m profundidad en el estero Elefantes. Anteriormente, se había registrado sobre rocas y algas a profundidades entre 0 y 10 m (Schrödl, 1996).

Distribución geográfica: En este crucero fue recolectado en la estación 25. Anteriormente, fue registrada desde Callao, Perú hasta Faro Corona, Chile; en el Atlántico hasta los $42^{\circ} 38^{\prime}$ S, Punta Pardelas, Valdez en la costa argentina (Schrödl, 1996).

Observaciones: Por primera vez se registra a una profundidad de $60 \mathrm{~m}$. La asignación de la especie al género Diaulula corresponde a una comunicación personal del Dr. M. Schrödl.

\section{BIVALVIA}

\section{SOLEMYIDAE}

\section{Acharax patagonica (E.A. Smith, 1885)}

(Fig. 3a)

Solemya patagonica E.A. Smith, 1885: 208-209, lám. 11, figs. 1, 1 a.

Solemya (Acharax) patagonica (Dall, 1908a): 2.

Acharax patagonica (Soot-Ryen, 1959): 20. Villarroel \& Stuardo, 1998: 160.

Solemya macrodactila Rochebrune \& Mabille, 1889: H109-110, lám. 8, fig. 4a, b.

Solenomya macrodactyla (Carcelles, 1950): 74, lám. 3, fig. 64.

Acharax macrodactila (Soot-Ryen, 1959): 20; Forcelli, 2000: 142, fig. 451.
Descripción: concha gruesa, casi en forma de paralelogramo. Extremo anterior algo más angosto que el posterior, el cual es truncado redondeado y ancho. Área del ligamento angosta. Periostraco grueso, rojo negruzco, con prolongaciones anchas y largas, con surcos anchos y oblicuos. Umbos amarillentos, lisos. Concha de color violáceo pálido, estriada con líneas rojas desordenadas. Talla máxima fue de $55 \mathrm{~mm}$.

Hábitat: las valvas se recolectaron a $237 \mathrm{~m}$ de profundidad.

Distribución geográfica: se recolectó en la estación 41. Anteriormente, Smith (1885) describió esta especie en los 52 $45^{\prime} 30^{\prime}$ 'S, $73^{\circ} 46^{\prime} \mathrm{W}$. También fue registrada en bahía Orange (Rochebrune \& Mabille, 1889) y para el norte de la isla de Chiloé (Dall, 1908b).

Observaciones: especie poco común o escasa. Un solo individuo fue colectado, con las valvas vacías pero unidas por el ligamento y en buenas condiciones. Forcelli (2000) muestra un ejemplar de $60 \mathrm{~mm}$. La sinonimia de A. macrodactila con A. patagonica fue sugerida por Dall (1908a) y Soot-Ryen (1959), y confirmada por Dell (1995). Forcelli (2000) ilustró una especie con una línea externa más oval como A. patagonica, pero el examen del ejemplar tipo en el British Museum (Natural History) indica que la especie de Smith es tan truncada como la descrita por Rochebrune \& Mabille (1889). Villarroel \& Stuardo (1998) muestran un ejemplar de Acharax sp. de $2,7 \mathrm{~mm}$ de longitud, que podría ser un juvenil de esta especie.

\section{NUCULIDAE}

Ennucula puelcha (D'Orbigny, 1842) (Fig. 2j)

Nucula puelcha D’Orbigny, 1842: 162; D’Orbigny, 1846: 624, Atlas (1842) lám. 84, figs. 24-26 (as puelchana); Carcelles, 1944: 268; Castellanos, 1967: 189, lám. 14, fig. 5.

Ennucula puelcha (Dell, 1964b): 141; Bernard, 1983: 10; Villarroel \& Stuardo, 1998: 134-136 (sinonimia), figs. 29-32, 72, 110-112.

Descripción: concha gruesa, inflada, de forma oval trígona, superficie lisa con líneas de crecimiento marcadas. Lúnula elevada y levemente delimitada. Periostraco brillante, amarillo verdoso y más oscuro en los bordes. Umbos ubicados en el tercio posterior de la concha. Borde posterior muy corto. Lado dorsal suavemente arqueado. Lado ventral convexo 
y borde liso. Charnela con fosa del ligamento evidente, angosto, con menos dientes posteriores que anteriores. Talla máxima de $18,8 \mathrm{~mm}$ de longitud.

Hábitat: en este estudio los ejemplares fueron recolectados entre los 166 y $330 \mathrm{~m}$. Anteriormente, Villarroel \& Stuardo (1998) registran esta especie en Chile central entre 50 y $295 \mathrm{~m}$ de profundidad, en sustrato de fango, fango arenoso, arena fina, piedrecillas, junto a E. grayi, Tindaria virens y Yoldiella chilenica. Castellanos (1967) indica su registro a $63 \mathrm{~m}$ de profundidad sobre fondo arenoso.

Distribución geográfica: se recolectó en las estaciones $6,16,17,21,22,29,32,35,37$ y 39. Se ha registrado también en la costa uruguaya y bonaerense, hasta bahía San Blas (Castellanos, 1967). Extremo oriental del canal Beagle e isla Wollaston, estrecho de Magallanes, golfo de Ancud, Mehuin hasta punta Aguja, Perú (Villarroel \& Stuardo, 1998).

Observaciones: los 30 ejemplares fluctuaron entre 9,3 y 18,8 mm de longitud. Para la identificación de esta especie se ha seguido a Villarroel \& Stuardo (1998). Sin embargo, de acuerdo a D’Orbigny (1846: 624) la especie tiene un ángulo apical de $105^{\circ}$ (como fue confirmado en los tipos posibles, BMNH 1854.12.4.774) y es más truncada posteriormente que los ejemplares presentes en la muestra, sugiriendo que la taxonomía de esta especie requiere una revisión.

\section{Ennucula grayi (D'Orbigny, 1846) (Fig. 2h)}

Nucula grayi D’Orbigny, 1846: 625.

Ennucula grayi (Soot-Ryen, 1959): 13, lám. 1, fig.3; Ramorino, 1968: 186-189, lám. 1, fig. 2, lám. 4, fig. 4; Villarroel \& Stuardo, 1998: 133 (sinonimia), figs. $8,70,71,73,107-109$.

Descripción: concha de regular tamaño, oblicuamente ovalada. La superficie es lisa, con finas líneas de crecimiento, cubierta por un periostraco brillante de color verde oliva a café negruzco. El extremo posterior es más obtuso que el anterior; el margen dorsal anterior curvo y el margen dorsal posterior recto. Umbos levemente inflados. Lado anterior casi el doble más largo que el posterior. Charnela con 9 a 19 dientes anteriores y 3 a 9 posteriores. Condróforo oblicuo. La talla máxima controlada fue de 19,7 mm de longitud.

Hábitat: en este estudio los ejemplares se recolectaron entre 200 y 237 m de profundidad. La expedición Sueca (1948-49) encontró ejemplares entre 35 y $250 \mathrm{~m}$, algunas valvas fueron obtenidas a $500 \mathrm{~m}$ de profundidad, en sedimentos que varían entre arena y arcilla (Soot-Ryen, 1959). En Valparaíso sólo en sedimentos de arena y fango, entre 60 y $138 \mathrm{~m}$ (Ramorino, 1968). Posteriormente, Villarroel \& Stuardo (1998) la capturan a 94 y 772 m de profundidad en arena fango, pero la especie es más abundante entre los 180 y $221 \mathrm{~m}$ de profundidad.

Distribución geográfica: los ejemplares se recolectaron en las estaciones 10, 21 y 41. Anteriormente, fue descrita desde $45^{\circ} \mathrm{S}$ en la costa Atlántica hasta el estrecho de Magallanes y en la costa de Chile, hasta Coquimbo $31^{\circ} 51^{\prime}$ S (Villarroel \& Stuardo, 1998).

Observaciones: esta especie tiene un amplio rango batimétrico. Ramorino (1968) en la bahía de Valparaíso registró tallas entre 5,6 y 19,0 mm, en este estudio las tallas variaron entre 9,5 y $19,7 \mathrm{~mm}$.

\section{TINDARIIDAE}

\section{Tindariopsis sulculata Couthouy in Gould, 1852 (Fig. 2k)}

Nucula striata King \& Broderip, 1832: 337 (non Lamarck, 1805).

Tindaria striata (Carcelles, 1950): 73, lám. 3, fig. 63; Castellanos, 1967: 194, lám. 14, fig. 4; Forcelli, 2000: 143, fig. 457.

Tindaria sulculata Gould, 1852: 424-425, Atlas lám. 37, fig. 539a-c.

Tindaria (Tindariopsis) sulculata (Soot-Ryen, 1959): 19 (sinonimia).

Tindariopsis sulculata (Villarroel \& Stuardo, 1998): 144-145 (sinonimia)

Descripción: la concha es sólida, triangular con el lado posterior agudo. Los umbos son salientes y levemente prosogiros. Lúnula definida. Superficie exterior con fuertes estrías concéntricas regulares. Con periostraco de color café verdoso, oliváceo. Charnela con 11 dientes angulosos anteriores y 16 dientes angulosos posteriores. Seno paleal regularmente desarrollado. Ligamento exterior y pequeño. Exterior con fuertes estrías concéntricas regulares. Talla máxima controlada fue de 9,7 mm.

Hábitat: los ejemplares se obtuvieron a $237 \mathrm{~m}$ de profundidad junto con el braquiópodo Macandrevia sp. Previamente, fue registrada para el litoral de la costa Argentina entre 60 y 120 m (Castellanos, 1967). 
Distribución geográfica: se recolectaron ejemplares en las estaciones 14 y 41 . Anteriormente, fue descrita desde el río de La Plata hasta la región magallánica y Chile sin precisar localidad (Castellanos, 1967). Soot-Ryen (1959) registra esta especie para muchas estaciones del seno de Reloncaví y golfo de Ancud, y Carcelles \& Williamson (1951) registran esta especie para el norte de Talcahuano.

Observaciones: Villarroel \& Stuardo (1998) indican sólo a Tindaria virens y T. salaria para las costas de Chile.

Tindaria virens (Dall, 1890) (Fig. 2i)

Malletia (Tindaria) virens Dall, 1890: 254, lám. 13, fig. 3.

Tindaria virens (Soot-Ryen, 1959): 18-19; Villarroel \& Stuardo 1998: 158 (sinonimia), figs. 52, 54, 55, 93-96, 119, 120.

Descripción: concha pequeña (hasta $6 \mathrm{~mm}$ ), triangular, gruesa, inflada, con el lado anterior más corto que el posterior. Con periostraco amarillento, verdoso o pardo claro. Margen dorsal posterior obliquo, casi recto. Escultura externa formada por costillas concéntricas muy regulares y notorias. El color es blanco. La charnela con numerosos dientes en forma de $\mathrm{V}$ invertida, los posteriores en mayor número (hasta 18), que los anteriores (hasta 13).

Hábitat: En este estudio la especie fue recolectada a $250 \mathrm{~m}$ de profundidad. Anteriormente Soot-Ryen (1959) la recolectó sobre sustrato de fango, arcilla, arena con arcilla entre 250 a $300 \mathrm{~m}$. También fue registrada entre 204 y $750 \mathrm{~m}$ por Dall (1908b). La última mención de Villarroel \& Stuardo (1998) indica que la especie se encontró entre 70 y $460 \mathrm{~m}$ profundidad, en sustrato fangoso.

Distribución geográfica: en este estudio se registró en la estación 37. Soot-Ryen (1959) indica que se distribuye en la región Magallánica, seno de Reloncaví y parte norte del golfo de Ancud. Villarroel \& Stuardo (1998), señalan que se encuentra desde el golfo de Ancud al estrecho de Magallanes.

Observaciones: es una especie rara y parece confinada a áreas de profundidades superiores a $70 \mathrm{~m}$ (Soot-Ryen, 1959).

\section{LIMOPSIDAE}

\section{Limopsis marionensis E.A. Smith, 1885}

(Fig. 3b)

Limopsis marionensis E.A. Smith, 1885: 254, pl.18, figs. 2-26; Dell, 1990: 19 (sinonimia), figs. 25-26.

Descripción: la concha es redondeada oblicua, con un denso periostraco piloso de color café oscuro. Umbos pequeños y agudos separados por el área del ligamento que es estrecha. La superficie externa con estrías concéntricas y radiales muy finas. Color blanco. La charnela es angosta ligeramente curva con numerosos dientes a cada lado de la foseta. El interior es de color blanco, con la impresión del músculo anterior pequeño y posterior más grande y piriforme. Talla máxima controlada de $26 \mathrm{~mm}$ de longitud.

Hábitat: En este estudio se encontró sobre fondo arenoso a $250 \mathrm{~m}$ profundidad junto con Pandora cistula, Ennucula puelcha, Cyclopecten vitreus, Falsilunatia patagonica y Cyclocardia velutina. Anteriormente fue registrada sobre fondos blandos con fragmentos de tubos de poliquetos entre $250 \mathrm{y}$ $300 \mathrm{~m}$ de profundidad (Soot-Ryen, 1959). Posteriormente, fue citada por Stuardo (1962) a $800 \mathrm{~m}$ de profundidad frente a la costa central de Chile. Dell (1990) indica que en el mar de Ross vive por encima de los $1.674 \mathrm{~m}$.

Distribución geográfica: en este crucero se registró en la estación 32. Se distribuye desde el seno de Reloncaví a la región magallánica; costa del Atlántico, islas Malvinas y alrededor de las región antártica, circumpolar antártica excepto Georgia del sur (Dell, 1990). Stuardo (1962) la registra frente a la costa de Algarrobo ( $33^{\circ} 20^{\prime} \mathrm{S}$ ).

Observaciones: es una especie rara que vive a profundidades mayores de $250 \mathrm{~m}$. Sin embargo, tiene una amplia distribución geográfica. Es la especie más grande del género y sobrepasa los $80 \mathrm{~mm}$ (Dell, 1990).

\section{PECTINIDAE}

Cyclopecten vitreus (Gmelin, 1791) (Fig. 3d)

Ostrea vitrea Gmelin, 1791: 3328.

Cyclopecten (Delectopecten) vitreus (Grau, 1959): 47-50 (sinonimia), lám. 18.

Cyclopecten vitreus (Bernard, 1983): 26; Forcelli, 2000: 153, fig. 498. 


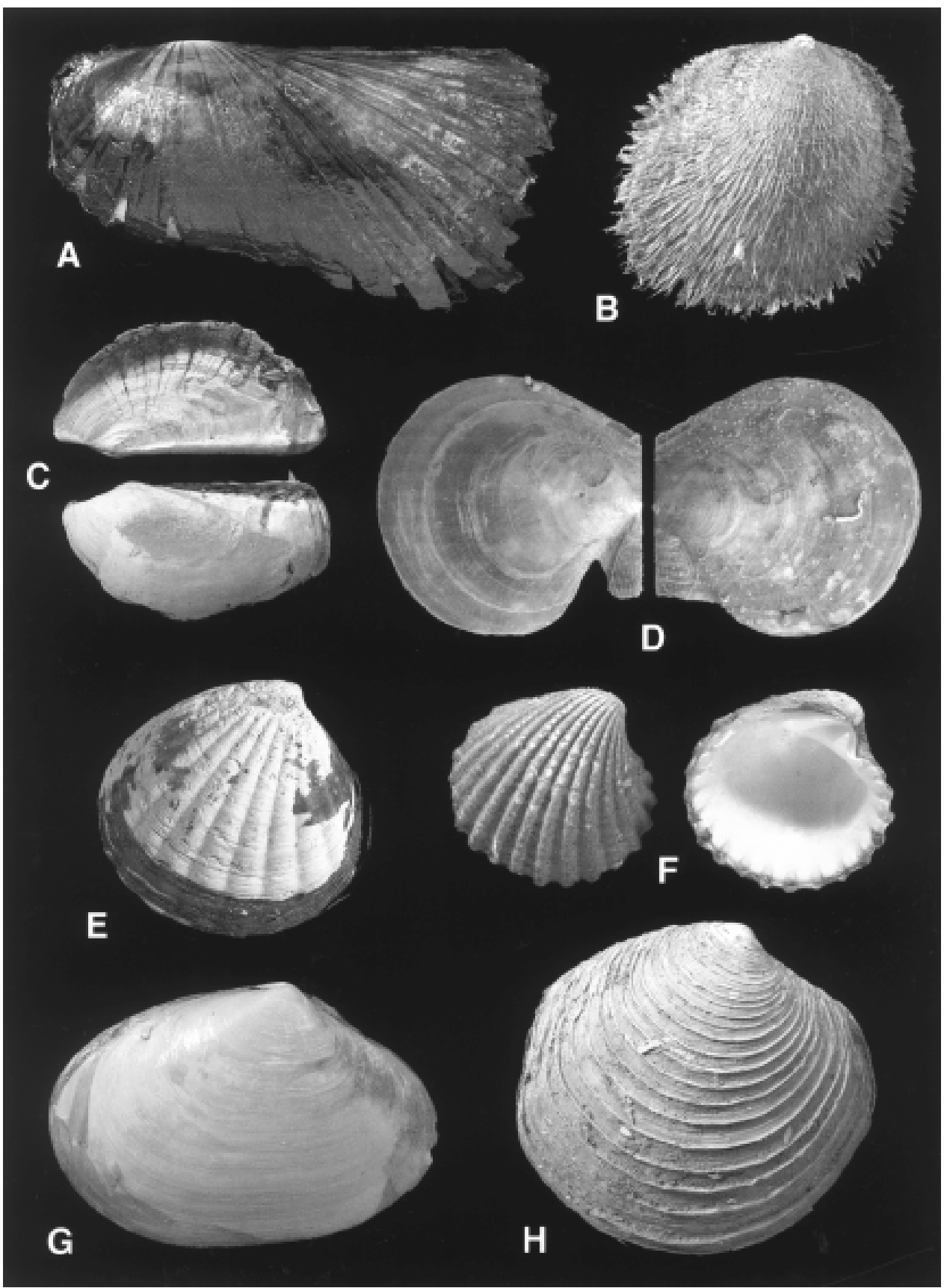

Figura 3. Lista de especies fotografiadas. a) Acharax macrodactila (Rochebrune \& Mabille, 1891), estación 41, 55 mm, b) Limopsis marionensis (E.A. Smith, 1885), estación 32, 21 mm, c) Pandora cistula Gould, 1850, estación 17A, 17 mm, d) Cyclopecten vitreus (Gmelin, 1791), estación 32, 17 mm, e) Cyclocardia compressa (Reeve, 1843), estación 26, 13,9 mm, f) Cyclocardia velutina (E.A. Smith, 1881), estación 14, 9,8 mm g) Macoma inornata (Hanley, 1844), estación 35, 18 mm h) Lucinoma antarctica (E.A. Smith, 1881), estación 17A, 22 mm.

Figure 3. List of species photographied. a) Acharax macrodactila (Rochebrune \& Mabille, 1891), station 41, 55 mm, b) Limopsis marionensis (E.A. Smith, 1885), station 32, 21 mm, c) Pandora cistula Gould, 1850, station 17A, $17 \mathrm{~mm}, \mathrm{~d})$ Cyclopecten vitreus (Gmelin, 1791), station 32, $17 \mathrm{~mm}$, e) Cyclocardia compressa (Reeve, 1843), station 26, 13.9 mm, f) Cyclocardia velutina (E.A. Smith, 1881), station 14, $9.8 \mathrm{~mm}, \mathrm{~g}$ ) Macoma inornata (Hanley, 1844), station 35, 18 mm, h) Lucinoma antarctica (E.A. Smith, 1881), station 17A, 22 mm. 
Descripción: concha de forma subcircular, pequeña, moderadamente convexa, frágil, delgada, semi transparente. Con los bordes simples y lisos. Orejas muy desiguales, anteriores más largas que las posteriores, con finas, pero nítidas costillas radiales escamosas. La escultura de ambas valvas con finas y regulares estrías concéntricas y radiales irregulares, poco notorias. Sin embargo son diferentes en escultura y tamaño, la valva derecha es más pequeña y con la escotadura bisal. Color blanquizco. El tamaño máximo de la concha fue de $17 \mathrm{~mm}$.

Hábitat: en este estudio se localizó a $250 \mathrm{~m}$ de profundidad. Grau (1959) indica que ha sido encontrado sobre fondos de fango a profundidades de 27 a $4312 \mathrm{~m}$ a menudo adherido a Hydroida y Gorgonacea; los especímenes japoneses frecuentemente se encuentran adheridos al crustáceo Macrocheira kaempferi De Haan. El género está ampliamente distribuido a grandes profundidades.

Distribución geográfica: en este crucero se registraron ejemplares en la estación 32. La especie tiene un amplio rango de distribución geográfica en muchos lugares del mundo, en Sudamérica se registró en Santa Cruz, Argentina, estrecho de Magallanes y sur de Chile (Grau, 1959).

Observaciones: "este es el Pectínido más ampliamente distribuido en el mundo" (Grau, 1959).

\section{LUCINIDAE}

\section{Lucinoma lamellata (E.A. Smith, 1881)}

(Fig. 3h)

?Lucina antarctica Philippi, 1855: 209.

Lucinoma antarctica (Bernard, 1983): 29; Forcelli, 2000: 154, fig. 499.

Diplodonta lamellata E.A. Smith, 1881: 38, lám. 15, fig. 1a-c.

Lucinoma lamellata (Carcelles, 1950): 79, lám. 4, fig. 81; Soot-Ryen, 1959: 41, lám. 1, fig. 12.

Descripción: concha grande, subcircular, los umbos son pequeños. La escultura con lamelas concéntricas sobresalientes. El periostraco débil, de color café amarillento, la concha de color blanco. Lúnula angosta. La charnela con dos dientes cardinales divergentes en cada valva, dientes anterior y posterior lateral lamelados en la valva derecha, que se corresponde a surcos en la izquierda. Línea paleal entera, a veces granular, estriada o punctada. Tamaño máximo de $50 \mathrm{~mm}$.
Hábitat: los ejemplares se localizaron a profundidades entre 166 y 330 m, junto con Ennucula grayi, Pandora cistula, Fissidentalium majorinum y Antalis perceptum. Anteriormente fueron registrados pequeños ejemplares a profundidades entre 260 y $500 \mathrm{~m}$ sobre fondo de fango fino y arcilla (SootRyen, 1959).

Distribución geográfica: en este estudio la especie se registró en las estaciones 6, 16, 17, 22, 37 y 41. Anteriormente, fue mencionada para el seno de Reloncaví, sur de Chile, Magallanes y banco Burdwood (Soot-Ryen, 1959).

Observaciones: los ejemplares recolectados tienen tamaños que varían entre 18 a $21,2 \mathrm{~mm}$ de longitud. La nomenclatura utilizada sigue a Soot-Ryen (1959). De acuerdo a Bernard (1983) Lucina antarctica Philippi, 1855 es el primer nombre, pero esta especie no fue dibujada y no existe el tipo disponible.

\section{CARDITIDAE}

\section{Cyclocardia compressa Reeve, 1843 (Fig. 3e)}

Cardita compressa Reeve, 1843: 194.

Cyclocardia compressa (Soot-Ryen, 1959): 39; Dell, 1964b: 190-191 (sinonimia); Ramorino, 1968: 198201; Bernard, 1983: 34-35; Forcelli, 2000: 160, fig. 527.

Descripción: concha algo triangular, ovalada, oblicua, posteriormente angulada y aplanada. Color blanco con periostraco de color amarillo a café rojizo. La superficie externa ornamentada con 13 a 18 costillas radiales anchas, planas, más o menos lisas y separados por surcos angostos. Umbos prominentes, subcentrales, prosogiros. Charnela de la valva izquierda con 2 dientes cardinales; valva derecha con un diente central grueso, un pequeño diente anterior, y una lamina posterior paralela al ligamento; dientes laterales vestigiales, uno anterior y uno posterior en cada valva. Superficie interna reflejando claramente la escultura externa de la concha. La talla máxima fue de 13,9 $\mathrm{mm}$.

Hábitat: los ejemplares se localizaron entre $20 \mathrm{y}$ $76 \mathrm{~m}$ de profundidad, junto al braquiópodo Novocrania lecointei, nudibranquios y Zygochlamys patagonica.

Distribución geográfica: en este estudio los ejemplares se recolectaron en las estaciones 13 y 26. La distribución geográfica conocida para esta especie es de Valparaíso a Magallanes (Soot-Ryen, 1959). 
Posteriormente, Ramorino (1968) la indicó de Arica a Magallanes, e islas Malvinas.

Observaciones: esta especie fue registrada en la bahía de Valparaíso a $65 \mathrm{~m}$ de profundidad en fondos de arena y las tallas variaron entre 2,7 y $11,4 \mathrm{~mm}$ de longitud (Ramorino, 1968).

\section{Cyclocardia velutinus (E.A. Smith, 1881)} (Fig. 3f)

Cardita (Actinobolus) velutinus E.A. Smith, 1881: 42, lám. 5, fig. 8.

Cyclocardia velutina (Soot-Ryen, 1959): 39; Dell, 1964b: 191 (sinonimia).

Cyclocardia velutinus (Barnard, 1983): 35; Forcelli, 2000: 160, fig. 529.

Descripción: concha gruesa, de color blanco con periostraco grueso de color pardo verdoso. Lúnula marcada profundamente por una línea cordiforme. Exteriormente con costillas elevadas y nodulosas, por las estrías de crecimiento. Charnela de la valva derecha con un diente cardinal central, valva izquierda con dos fuertes dientes cardinales. Interior de color blanquizco azul, con pintas de color amarillento bajo el umbo. Talla máxima de 14,4 mm longitud.

Hábitat: los ejemplares se registraron entre $76 \mathrm{y}$ $250 \mathrm{~m}$ de profundidad, junto a los braquiópodos Novocrania lecointei y Macandrevia sp. Anteriormente fue encontrada sobre arena con conchas y pequeñas piedras. Ejemplares vivos se encontraron a profundidades entre 20 y $45 \mathrm{~m}$, y solo valvas a los 250 m (Soot-Ryen, 1959).

Distribución geográfica: en este estudio la especie se encontró en las estaciones 13, 14 y 32. La distribución geográfica se indica para Arica a Magallanes (Soot-Ryen, 1959). Costa de la Patagonia, islas Malvinas (Dell, 1964b).

Observaciones: la identificación fue confirmada por comparación con los tipos (BMNH 1879.10.15.125146).

\section{TELLINIDAE}

Macoma inornata (Hanley, 1844) (Fig. 3g)

Tellina inornata Hanley, 1844: 44.

Macoma (Psammacoma) inornata (Soot-Ryen, 1959): 62-63, lám. 4, figs. 34-36; Dell, 1964b: 220 (sinonimia), lám. 7, figs. 1, 2, 4; Ramorino, 1968: 220-223 (sinonimia), lám.3, fig. 5, lám. 10, figs. 1, 2; Dell, 1971: 177; Bernard, 1983: 45.

Macoma inornata (Forcelli, 2000): 163, fig. 546.

Descripción: valvas delgadas de forma oblonga triangular, aplanada, con el lado anterior más largo y la parte posterior levemente flectada hacia la derecha. La superficie es de color blanco tiza, con manchas café oxido o depósitos terrosos color plomo, con un periostraco de color grisáceo presente sobre los bordes de las valvas; presenta además finas estrías concéntricas de crecimiento microscópicamente laminares y con una cresta o hendidura que corre desde el umbo al extremo posterior, también se observan débiles líneas radiales. Ligamento notorio de color café oscuro. Charnela con dos dientes cardinales en cada valva; el anterior izquierdo y posterior derecho bífidos en sus ápices. Seno paleal profundo, en la valva izquierda poco más grande que en la derecha. La talla máxima fue de $22,3 \mathrm{~mm}$ de longitud.

Hábitat: en este estudio se encontró entre 106 y $200 \mathrm{~m}$ de profundidad junto con el braquiópodo Macandrevia sp. Anteriormente, fue registrado sobre arena y arcilla mezclada con fango, entre 15 y $300 \mathrm{~m}$ de profundidad (Soot-Ryen, 1959).

Distribución geográfica: los ejemplares se recolectaron en las estaciones 14, 21, 29 y 35. Anteriormente, fue descrita desde Atacama al golfo de Ancud e islas Malvinas (Ramorino, 1968).

Observaciones: el tamaño de los ejemplares fluctuó entre 15 y $22,3 \mathrm{~mm}$ de longitud. Ramorino (1968) registró tamaños de $27 \mathrm{~mm}$ de longitud en ejemplares de la bahía de Valparaíso.

\section{PANDORIDAE}

Pandora cistula Gould, 1850 (Fig. 3c)

Pandora cistula Gould, 1850: 217; Carcelles, 1950: 83; Forcelli, 2000: 171, fig. 583.

Kennerlyia patagonica Dall, 1915: 450.

Pandora (Kennerlia) patagonica (Lamy, 1934): 110.

Kennerlia patagonica (Soot-Ryen, 1959): 37; Ramorino, 1968: 223-225.

Descripción: concha delgada, frágil, aplanada, transversalmente alargada. Umbos poco sobresalientes. Lado anterior corto y curvo, el posterior largo y truncado. Inequivalva; valva derecha plana a veces 
algo cóncava y en la zona central presenta líneas radiales irregulares de longitud variable que no alcanzan al umbo, la izquierda es convexa y provista de dos quillas oblicuas en su parte dorsal posterior. La superficie de ambas valvas tiene estrías finas concéntricas de crecimiento. Color beige, pero la zona umbonal es blanca opaca, por pérdida de periostraco blanquizco, membranoso y quebradizo. La talla máxima fue de 17,8 $\mathrm{mm}$ de longitud.

Hábitat: los ejemplares se registraron entre 166 y $330 \mathrm{~m}$ de profundidad, junto al braquiópodo Macandrevia sp. Anteriormente, se encontró a 50 $\mathrm{m}$ de profundidad, en sustrato tipo fango arena con fragmentos de conchas (Soot-Ryen, 1959). Ejemplares vivos fueron recolectados en arena fangosa y fango arenoso en la bahía de Valparaíso (Ramorino, 1968).

Distribución geográfica: los ejemplares se colectaron en las estaciones 4, 14, 16, 17, 20, 21, 22, 32 y 41. Anteriormente, Soot-Ryen (1959) la menciona desde el sur de Chile al norte del estero Reloncaví. Luego, Ramorino (1968) amplió su distribución a la bahía de Valparaíso.

Observaciones: los tamaños registrados variaron entre 8,3 y $17,8 \mathrm{~mm}$ de longitud. Estas tallas son mayores que las registradas para los ejemplares de la bahía de Valparaíso, que fluctuaron entre 3,5 y 12 $\mathrm{mm}$.

\section{SCAPHOPODA \\ DENTALIIDAE}

\section{Fissidentalium majorinum (Mabille \& Rochebrune, en Rochebrune \& Mabille, 1889) (Fig. 2f)}

Dentalium majorinum Rochebrune \& Mabille, 1889: H100, lám. 4, fig. 10; Castellanos, 1979: 133; Forcelli, 2000: 172, fig. 586.

Dentalium (Fissidentalium) majorinum (Dell, 1964b): 123 (sinonimia).

Fissidentalium majorinum (Dell, 1990): 72 (sinonimia).

Descripción: concha tubular muy larga, grande, gruesa, levemente curvada, con fuertes costillas longitudinales. Aproximadamente 11 costillas cerca del ápice, separadas con intervalos cóncavos, el número aumenta a cerca de 29 en la apertura. Las costillas hacia el ápice están bien marcadas y regu- larmente alternadas, pero llegan a ser subiguales hacia la abertura, y separadas por surcos cóncavos más anchos donde ocasionalmente se observan estrías secundarias débiles. La superficie también presenta finas estrías transversales de crecimiento, las cuales son más visibles en los intervalos. El orificio anal es subcircular o biselado y no presenta hendidura ni incisión. Los ejemplares presentan 2 a 4 profundos surcos concéntricos a diferentes alturas de su longitud. La longitud máxima fue de 39,5 mm.

Hábitat: en este estudio se encontraron en arena entre 200 y $250 \mathrm{~m}$ de profundidad, junto con ejemplares de Ennucula puelcha, Nassarius gayii, Macoma inornata y Pandora cistula. En el mar de Ross, se capturó entre 328 y 836 m de profundidad (Dell, 1990).

Distribución geográfica: se registraron ejemplares en las estaciones 10, 16, 21 y 22. La literatura indica que la especie es circumpolar antártica y se distribuye entre $\operatorname{los} 49^{\circ}$ y $145^{\circ} \mathrm{E}$ en el continente sudamericano, islas Malvinas, Shetlands del sur y región Magallánica. El presente registro extiende hacia el norte la distribución geográfica.

Observaciones: los ejemplares midieron entre 24,4 y $39,5 \mathrm{~mm}$ de longitud. Los verdaderos Fissidentalium presentan una profunda y angosta hendidura. Sin embargo, nuestros ejemplares se caracterizan por la ausencia de hendidura apical, similar observación fue percibida por Dell (1964b) para los especímenes magallánicos, quien además indica que es tal vez el molusco mejor documentado en su rango de distribución.

\section{Rhabdus perceptum (Mabille \& Rochebrune, en Rochebrune \& Mabille, 1889) (Fig. 2g)}

Dentalium perceptum Rochebrune \& Mabille, 1889: H99; Carcelles, 1950: 73; Carcelles \& Williamson, 1951: 322.

Rhabdus perceptum (Steiner \& Kabat, 2001): 449.

Descripción: concha muy alargada, tubular, grande, levemente curvada. Superficie externa lisa con estrías concéntricas finas y regulares, estrías longitudinales muy débiles. Con el ápice en forma circular sin hendidura ni tapón. La talla máxima de los ejemplares fue de $43 \mathrm{~mm}$ de longitud.

Hábitat: los ejemplares se recolectaron entre 220 y $250 \mathrm{~m}$ de profundidad, junto a Fissidentalium majorinum, Nassarius gayii y Ennucula puelcha. 
Distribución geográfica: en este estudio se encontraron ejemplares en las estaciones 10,16 y 21 . Anteriormente, esta especie fue descrita para la región Magallánica y sur de Patagonia (Carcelles, 1950). El presente registro extiende hacia el norte la distribución geográfica.

Observaciones: la descripción del género indica que las especies presentan sobre el lado convexo una hendidura en forma de $\mathrm{V}$ y un sólido o grueso tapón, desde el cual emerge una delgada o angosta pipa central, estas estructuras no se observan en los ejemplares recolectados.

\section{DISCUSIÓN}

El total del material identificado en el área fue de 48 especies, de las cuales 5 están en estudio y 21 ya fueron descritas por Reid \& Osorio (2000). En este trabajo se agrega la descripción de 22 especies, con nuevos datos del hábitat y de distribución geográfica, señalándose los nuevos registros geográficos. De las 21 especies comunes en ambos estudios (Tabla 2), los poliplacóforos mantienen el mismo rango de profundidad (0 a $15 \mathrm{~m}$ ), pero la mayoría (17 especies) aumenta notoriamente sus rangos batimétricos. En algunas especies sólo sus valvas fueron registradas en buenas condiciones. Es posible que se desprendieran desde la superficie y cayeran al fondo, otras fueron encontradas vivas como es el caso de Nassarius gayii, Adelomelon ancilla, Tritonia challengeriana y Zygochlamys patagonica. La presencia sólo de poliplacóforos en el intermareal, indicaría que allí se obtuvo una muestra sesgada. La ausencia de micromoluscos y de moluscos de talla pequeña se debería al uso de una metodología inadecuada, ya que la rastra Agassiz limita la captura de micromoluscos.

Tabla 2. Nuevos registros de profundidades para los moluscos recolectados en el estero Elefantes y áreas adyacentes.

Table. 2. New depth records of molluscs recorded in Elefantes fjord and adjacent areas.

\begin{tabular}{|lccc|}
\hline Especie & $\begin{array}{c}\mathbf{2 0 0 0} \\
\text { Profundidad (m) }\end{array}$ & $\begin{array}{c}\mathbf{2 0 0 1} \\
\text { Profundidad (m) }\end{array}$ & Observaciones \\
\hline Plaxiphora aurata & $0-5$ & $0-5$ & vivo \\
Chiton granosus & 0 & $0-5$ & vivo \\
Tonicia atrata & 0 & $0-5$ & vivo \\
Tonicia chilensis & $10-15$ & $0-5$ & vivo \\
Iothia coppingeri & $10-15$ & 106 & valvas \\
Homalopoma cunninghami & $10-15$ & $76-160$ & valvas \\
Crepidula dilatata & $0-15$ & 20 & valvas \\
Xymenopsis muriciformis & $6-15$ & 20 & valvas \\
Pareuthria fuscata & 0 & 166 & valvas \\
Nassarius gayii & $6-15$ & $166-250$ & vivo \\
Adelomelon ancilla & 10 & 204 & vivo \\
Tritonia challengeriana & 0 & 106 & vivo \\
Chilina patagonica & 0 & 200 & valvas \\
Nucula pisum & $10-15$ & 106 & valvas \\
Mytilus edulis chilensis & 0 & 330 & valvas \\
Choromytilus chorus & 0 & 160 & valvas \\
Zygochlamys patagonica & $10-15$ & $20-120$ & vivo \\
Carditopsis flabellum & $10-15$ & $76-250$ & vivo \\
Ensis macha & $5-15$ & 60 & valvas \\
Venus antiqua & $7-15$ & $20-60$ & valvo \\
Hiatella solida & $3-5$ & 20 & \\
\hline
\end{tabular}

*Reid \& Osorio (2000) 
Los nuevos registros de moluscos (este estudio agrega siete especies) para el área de fiordos estaría indicando los vínculos con la malacofauna magallánica que avanza desde la zona austral hacia el norte mediante las corrientes marinas de grandes profundidades de acuerdo a Brattsröm \& Johansen (1983) y que se ratificaría con el escafópodo Fissidentalium majorinum y el gastrópodo Calliostoma nudiusculum que se registraron a profundidades mayores de $100 \mathrm{~m}$. Por el contrario, los vínculos con la fauna de la zona norte se realizarían por medio de las corrientes superficiales que van desde la zona norte a sur del país; la única especie del área septentrional registrada fue Aeneator fontainei $(60 \mathrm{~m})$. Registros similares también se han observado en peces (Pequeño \& Lamilla, 1997).

Es así como la fauna malacológica sería el resultado de la confluencia de especies provenientes de las provincias malacológicas magallánica, antártica y peruviana; en consecuencia un área de transición faunística con el aporte regular de especies de las áreas adyacentes. Según Reid \& Osorio (2000) el área de estudio corresponde al extremo sur de la zona de transición, donde los moluscos tienen escasos datos de distribución y donde no se reconocen hasta el momento especies endémicas.

El sistema de fiordos es extraordinariamente dinámico como ha sido señalado y estas observaciones además estarían indicando que es necesario continuar los estudios de la sistemática de micromoluscos, de los bordes costeros entre 0 y 20 $\mathrm{m}$ de profundidad, mediante el uso de instrumentos de captura cualitativos y cuantitativos. Esto, con el objeto de conocer la calidad y cantidad de los componentes que estructuran la comunidades bentónicas que son la base de las cadenas tróficas, como también para completar el conocimiento de la biodiversidad de los fiordos en el sur de Chile.

\section{AGRADECIMIENTOS}

Los autores agradecen la identificación de los braquiópodos a la Dra. Sarah Long (BMNH), de los nudibranquios al Dr. Michael Schröld, Zoologishe Staatssamlung München, Alemania, de los escafópodos al Dr. Victor Scarabino. El valioso trabajo de fotografía al Sr. H. Taylor del BMNH. La inestimable discusión sobre las especies de Calliostoma al Dr. B.A. Marshall y Lucinoma al Dr. J.D.Taylor del BMNH. A la revisión del texto a la Dra. S. Williams del BMNH. La colaboración téc- nica de la Srta. L. Ramajo. Las sugerencias y correcciones al manuscrito de los revisores que permitieron mejorar el texto. Agradecemos también el apoyo del Comité Oceanográfico Nacional (CONA), al Departamento de Ciencias Ecológicas de la Universidad de Chile, a la Comisión Nacional de Investigación Científica y Tecnología, Chile (CONICYT) y a la Royal Society de Inglaterra.

\section{REFERENCIAS}

Araya-Vergara, J. 1997. Perfiles geomorfológicos de los fiordos y depresión longitudinal de Norpatagonia. Cienc. Tecnol. Mar, 20: 3-22.

Araya-Vergara, J. 1999. Perfiles longitudinales de fiordos de Patagonia Central. Cienc. Tecnol. Mar, 22: 3-30.

Avaria, S., D. Cassis, P. Muñoz \& P. Vera. 1997. Distribución del microfitoplancton marino en aguas interiores del sur de Chile en octubre de 1995. Cienc. Tecnol. Mar, 20: 107-124.

Avaria, S., L. Jorquera, P. Muñoz \& P. Vera. 1999. Distribución del microfitoplancton marino en la zona de aguas interiores comprendidas entre el golfo de Penas y el estrecho de Magallanes, Chile, en la primavera de 1996 (Crucero Cimar-Fiordo 2). Cienc. Tecnol. Mar, 22: 81-109.

Balbontín, F. \& R. Bernal. 1997. Distribución y abundancia del ictioplancton en la zona austral de Chile. Cienc. Tecnol. Mar, 20: 155- 164.

Basten, J. \& A. Clement. 1999. Oceanografía del estero de Reloncaví, X Región de Chile. Cienc. Tecnol. Mar, 22: 31-46.

Bernard, F.R. 1983. Catalogue of the living Bivalvia of the Eastern Pacific Ocean: Bering Strait to Cape Horn. Can. Special Publ. Fish. Aquat. Sci., 61: 1102.

Bernal, R. \& F. Balbontín. 1999. Ictioplancton de los fiordos entre el golfo de Penas y el estrecho de Magallanes y factores ambientales asociados. Cienc. Tecnol. Mar, 22: 143-154.

Bonert, A.C. 1997. Identificación y cuantificación de hidrocarburos clorados en sedimentos entre seno de Reloncaví y golfo Elefantes. Cienc. Tecnol. Mar, 20: 195-199. 
Bonert, A.C. \& R. Estrada. 2001. Hidrocarburos clorados en sedimentos del área Otway-Beagle. Cienc. Tecnol. Mar, 24: 41-45.

Brattström, H. \& A. Johanssen. 1983. Ecological and regional zoogeography of the marine benthic fauna of Chile. Report $\mathrm{N}^{\circ} 49$ of the Lund Univ. Chile Expedition 1948-1949. Sarsia, 68: 289-339.

Carcelles, A. 1944. Las especies del género Capulus Montfort, 1810 en aguas argentinas. Com. Zool. Mus. Hist. Nat. Montevideo, 1(9): 1-5.

Carcelles, A. 1950. Catálogo de los moluscos marinos de la Patagonia. Anal. Mus. Nahuel Huapi, 2: 41-99.

Carcelles, A. \& S.I. Williamson. 1951. Catálogo de los moluscos marinos de la provincia magallánica. Rev. Inst. Nac. Invest. Cienc. Nat. Zool., 2(5): 225383.

Castellanos, Z.A. 1967. Catálogo de los moluscos marinos bonaerenses. Anal. Com. Invest. Cient. La Plata, Argentina, 8: 9-364.

Castellanos, Z.A. 1979. Micromoluscos poco conocidos del sur argentino-chileno. Neotropica, 25(74): 133-140.

Castellanos, Z.A. 1988. Catálogo descriptivo de la malacofauna marina magallánica 1 . Com. Invest. Cient. Bs. As., 1: 1-41.

Castellanos, Z.A. 1989. Catálogo descriptivo de la malacofauna marina magallánica 4. Com. Invest. Cient. Bs. As., 3: 1-40.

Castellanos, Z.A. 1990a. Catálogo descriptivo de la malacofauna marina magallánica 5. Com. Invest. Cient. Bs. As., 5: 1-36.

Castellanos, Z.A. 1990b. Catálogo descriptivo de la malacofauna marina magallánica 6 . Com. Invest. Cient. Bs. As., 6: 1-36.

Castellanos, Z.A. \& N.A. Landoni. 1989. Catálogo descriptivo de la malacofauna marina magallánica 3. Com. Invest. Cient. Bs. As., 3: 1-40.

Dall, W.H. 1890. Scientific results of explorations by the U.S. Fish Commission Steamer Albatross. VII. Preliminary report on the collection of Mollusca and Brachiopoda obtained in 1887-'88. Proc. U.S. Nat. Mus., 12: 219-362.

Dall, W.H. 1908a. A revision of the Solenomyacidae. Nautilus, 22: 1-2.
Dall, W.H. 1908b. Reports...U.S. Fish Commission Steamer "Albatross". Reports on the Mollusca and Brachiopoda. Bull. Mus. Comp. Zool., 43: 205-487.

Dall, W.H. 1915. A review of some bivalves shells of the group Anatinacea from the west coast of America. Proc. U.S. Nat. Mus., 49: 441-456.

Dell, R.K. 1964a. The forms of Capulus known from New Zealand. Rec. Dominion Mus., 5(7): 49-50.

Dell, R.K. 1964b. Antarctic and subantarctic Mollusca: Amphineura, Scaphopoda and Bivalvia. Discovery Rep., 33: 93-250.

Dell, R.K. 1971. The marine Mollusca of the Royal Society Expedition to southern Chile, 1958-1959. Rec. Dominion Mus., 7(17): 155-233.

Dell, R.K. 1990. Antarctic Mollusca with special reference to the fauna of the Ross Sea. Bull. Roy. Soc. New Zealand, 27: 1-311.

Dell, R.K. 1995. New species and records of deepwater Mollusca from off New Zealand. Tuhinga: Rec. Mus. N.Z. Te Papa Tongarewa, 2: 1-26.

D'Orbigny, A. 1834-1847. Voyage dans l'Amérique Méridionale. Paleontologie, 3(4): 1-187; Mollusques, 5(3): 1-758.

Fierro, J., M. Bravo \& M. Castillo. 2000. Caracterización del régimen de mareas y corrientes a lo largo del canal de Moraleda (43⒌'S-45'17'S). Cienc. Tecnol. Mar, 23: 3- 14.

Forcelli, D.O. 2000. Moluscos magallánicos: guía de moluscos de Patagonia y sur de Chile. Vásquez Mazzini Editores, Buenos Aires, 200 pp.

Frembly, J. 1827. A description of several new species of chitones, found on the coast of Chili, in 1825; with a few remarks on the method of taking and preserving them. Zool. J., 3: 193-205.

Gmelin, J.F. 1791. Caroli a Linné...Systema Naturae per Regna Tria Naturae, 1(6): 3021-3910.

Gould, A.A. 1850. Descriptions of shells from the United States Exploring Expedition. Proc. Boston Soc. Nat. Hist., 3: 214-219.

Gould, A.A. 1852. Mollusca and shells. U.S. Exploring Expedition, 12: 1-498.

Grau, G. 1959. Pectinidae of the Eastern Pacific. Allan Hancock Pacific Expeditions, 23: 1-308. 
Hanley, S.C.T. 1844. Description of new species of the genus Tellina chiefly collected by H. Cuming, Esq. in the Philippine Islands and Central America. Proc. Zool. Soc. London, 1844: 59-64.

Kaas, P. \& R. Van Belle. 1998. Catalogue of living chitons (Mollusca, Polyplacophora). Backhuys, Leiden, 204 pp.

King, P.P. \& W.J. Broderip. 1832. Description of the Cirrhipeda, Conchifera and Mollusca in a collection formed by the officers of H.M.S. Adventure and Beagle employed between the years 1826 and 1830 in surveying the southern coasts of South America, including the Straits of Magellan and the coast of Tierra del Fuego. Zool. J., 5: 332-349.

Lamy, E. 1934. Coquilles marines recueillies par ME Aubert de la Rue dans l'Amerique du Sud. Bull. Mus. Nat. d'Hist. Nat., N.S., 6: 432-435.

Leloup, E. 1956. Reports of the Lund University Chile Expedition 1948-49, 27. Polyplacophora. Lunds Universitets Arsskrift, N.F.Avd.2, 52(15): 1-94.

Linse, K. 2002. The shelled magellanic Mollusca: with special reference to biogeographic relations in the Southern Ocean. Theses Zoologicae, 34:1-252.

Marín, V. \& L. Delgado. 2001. Taxocenosis de copépodos calanoideos en los canales magallánicos: un patrón anidado. Cienc. Tecnol. Mar, 24: 81- 89.

McLean, J.H. \& H. Andrade. 1982. Large archibenthal gastropods of central Chile: Collections from an expedition of the R7V Anton Bruun and the Chilean shrimp fishery. Contrib. Sci. Nat. Hist. Mus., Los Angeles County, 342: 1-20.

Mujica, R.A. \& M. Medina. 1997. Larvas de crustáceos decápodos de los canales australes de Chile (4130'-46²0'S). Cienc. Tecnol. Mar, 20: 147154.

Mujica, A. \& M. Medina. 2000. Distribución y abundancia de larvas de crustáceos decápodos en el zooplancton de los canales australes. Proyecto Cimar-Fiordo 2. Cienc. Tecnol. Mar, 23: 49-67.

Osorio, C., M.E. Ramírez \& M. Vega. 2002. Distribución y abundancia de macroorganismos del intermareal de isla Traiguén $\left(45^{\circ} \mathrm{S}, 73^{\circ} \mathrm{W}\right)$, estero Elefantes, Región de Aysén, Chile. Bol. Mus. Nac. Hist. Nat., Chile, 51: 175-184.
Palma, S. \& S. Rosales. 1997. Sifonóforos epipelágicos de los canales australes chilenos (4130' 46²0'S). Cienc. Tecnol. Mar, 20: 125-146.

Palma, S., R. Ulloa \& L. Linacre. 1999. Sifonóforos, quetognatos y eufáusidos de los canales australes entre el golfo de Penas y el estrecho de Magallanes. Cienc. Tecnol. Mar, 22: 111-142.

Pequeño, G. \& J. Lamilla. 1997. Peces litorales del crucero Cimar-Fiordo I. Cienc. Tecnol. Mar, 20: $165-174$

Pequeño, G. 1999. Peces del Crucero Cimar-Fiordo 2 a los canales patagónicos de Chile, con consideraciones ictiogeográficas. Cienc. Tecnol. Mar, 22: 165-179.

Pequeño, G. 2000. Peces del Crucero Cimar-Fiordo 3, a los canales del sur de Magallanes (CA. 55 ${ }^{\circ} \mathrm{S}$ ), Chile. Cienc. Tecnol. Mar, 23: 83-94.

Philippi, R.A. 1845. Diagnosen einiger neuen Conchylien, Archiv für Naturgeschichte, 11: 5071.

Philippi, R.A. 1855. Observaciones sobre las conchas de Magallanes. An. Univ. Chile, pp. 201-213.

Pineda, V., X. Contardo, G. Alfaro \& S. Helle. 2002. Caracterización textural, mineralógica y geoquímica de los sedimentos del canal Beagle y bahía Nassau, XII Región de Magallanes, Chile (crucero Cimar-Fiordo 3). Cienc. Tecnol. Mar, 25: 5-22.

Powell, A.W.B. 1951. Antarctic and subantarctic Mollusca: Pelecypoda and Gastropoda. Discovery Rep., 26: 47-196.

Ramorino, L. 1968. Pelecypoda del fondo de la bahía de Valparaíso. Rev. Biol. Mar., 13(3): 175-285.

Reeve, L.A. 1843-1878. Monograph of the genus Cardita. In: L.A Reeve (ed.). Conchologica Iconica. London, pp. 1-9.

Reid, D.G. \& C. Osorio. 2000. The shallow-water marine Mollusca of the Estero Elefantes and Laguna San Rafael, southern Chile. Bull. Nat. Hist. Mus. Zool., London, 66(2): 109-146.

Retamal, M. \& M. Gorny. 2001. Decápodos de los fiordos de Chile. Cienc. Tecnol. Mar, 24: 91-100.

Rochebrune, A.T. \& J. Mabille. 1889. Mollusques. Mission Scientifique du Cap Horn 1882-1883. Zool, 6: 1-129. 
Schrödl, M. 1996. Nudibranchia y Sacoglossa de Chile: morfología externa y distribución. Gayana Zool., 60(1): 17-62.

Slieker, F. 2000. Chitons of the world. An illustrated synopsis of recent Polyplacophora. Cupra Maritima, Rome, 154 pp.

Silva, N. \& C. Calvete. 2002. Características oceanográficas físicas y químicas de canales australes chilenos entre el golfo de Penas y el estrecho de Magallanes. Cienc. Tecnol. Mar, 25(1): 2388.

Silva, N. \& P. Ortiz. 2002. C y N, su distribución y estequiometría, en sedimentos superficiales de la región sur de la zona de fiordos y canales australes de Chile, $52^{\circ}-56^{\circ} \mathrm{S}$ (Crucero Cimar- Fiordo 3). Cienc. Tecnol. Mar, 25(1): 89-108.

Silva, N., C. Calvete \& H. Sievers. 1997. Características oceanográficas físicas y químicas de canales australes chilenos entre Puerto Montt y Laguna San Rafael. Cienc. Tecnol. Mar, 20: 23-106.

Silva, N., V. De Vidts \& J.I. Sepúlveda. 2001. Materia orgánica, $\mathrm{C}$ y $\mathrm{N}$ su distribución y estequiometria en sedimentos superficiales de la región central de los fiordos y canales australes de Chile (Crucero Cimar-Fiordo 2). Cienc. Tecnol. Mar, 24: 23-40.

Smith, E.A. 1881. Account of the zoological collections made during the survey of H.M.S. 'Alert' in the Straits of Magellan and on the coast of Patagonia. IV. Mollusca and Molluscoidea. Proc. Zool. Soc. London, 4: 22-44.

Smith, E.A. 1885. Report on the Lamellibranchiata collected by HMS Challenger during the years 1873-1876. Challenger Reports, Zool., 13: 1-341.
Smith, E.A. 1891. Description of new species of shells from the Challenger Expedition. Proc. Zool. Soc. London, 1891: 436-445.

Smith, E.A. 1915. Mollusca. British Antarctic "Terra Nova" Expedition 1910. Nat. Hist. Zool., 2: 61112.

Soot-Ryen, T. 1951. Antarctic Pelecypods. Scientific Results of the Norwegian Antarctic Expeditions 1927-1928, 32: 1-49.

Soot-Ryen, T. 1959. Pelecypoda. Rep. Lund University Chile Expedition 1948-49. Lunds Univ. Arsskr. N.F. 2, 55(6): 1-86.

Steiner, G. \& A.R. Kabat. 2001. Catalogue of supraspecific taxa of Scaphopoda (Mollusca). Zoosystema, 23: 433-460.

Stuardo, J. 1962. Sobre el género Limopsis y la distribución de L. jousseaumei (Mabille y Rochebrune, 1889) (Mollusca: Bivalvia). Gayana Zool., 6: 5-11.

Strebel, H. 1905. Beitrage zur Kenntnis der Mollusken Fauna der Magalhaen Provinz. II. Zoologische Jahrbucher. Abteilung für Systematik, Geographie und Biologie der Tiere, 21(suppl. 8): 121-166.

Vega, M., F. Rocha \& C. Osorio. 2000. Variaciones espaciales y temporales de paralarvas de cefalópodos en el canal de Moraleda $\left(43^{\circ} \mathrm{S}\right)$ sur de Chile. Cienc. Tecnol. Mar, 23: 69-82.

Villarroel, M. \& J. Stuardo. 1998. Protobranchia (Mollusca: Bivalvia) chilenos recientes y algunos fósiles. Malacologia, 40(1-2): 113-229. 